\title{
Hybrid Approach for Detection of Hard Exudates
}

\author{
Dr. H. B. Kekre \\ Computer Engineering \\ MPSTME, NMIMS University \\ Mumbai, India
}

\author{
Dr. Tanuja K. Sarode \\ Computer Engineering \\ TSEC \\ Mumbai, India
}

\author{
Ms. Tarannum Parkar \\ Computer Engineering \\ DBIT \\ Mumbai, India
}

\begin{abstract}
Diabetic Retinopathy is a severe and widely spread eye disease which can lead to blindness. Hence, early detection of Diabetic Retinopathy is a must. Hard Exudates are the primary sign of Diabetic Retinopathy. Early treatment of Diabetic Retinopathy is possible if we detect Hard Exudates at the earliest stage. The main concentration of this paper is to discuss techniques for efficient detection of Hard Exudates. The first technique, discusses Hard Exudates detection using mathematical morphology. The second technique, proposes a Hybrid Approach for Detection of Hard Exudates. This approach consists of three stages: preprocessing, clustering and post processing. In preprocessing stage, we resize the image and apply morphological dilation. The clustering stage applies LindeBuzo-Gray and $\boldsymbol{k}$-means algorithm to detect Hard Exudates. In post processing stage, we remove all unwanted feature components from the image to get accurate results. We evaluate the performance of the above mentioned techniques using the DIARETDB1 database which provides ground truth. The optimal results will be obtained when the number of clusters chosen is 8 in both of the clustering algorithms.
\end{abstract}

Keywords-Diabetic Retinopathy; Hard Exudates; Clustering; Mathematical Morphology

\section{INTRODUCTION}

The term Diabetic Retinopathy (DR) consists of two words Diabetes and Retinopathy. Diabetes mellitus or simply diabetes, is a disease in which a person has high blood sugar, either because of low insulin production or low response to insulin produced. Retinopathy literally means damage to the retina. Hence, DR is a widely spread eye disease which is regarded as the manifestation of diabetes on the retina. DR is of two types. The first type is Non Proliferative Diabetic Retinopathy. In this condition, retinal capillaries get damaged and microscopic leaks form in these vessels. Leakage causes the retina to swell, which interferes with normal vision. The second type is Proliferative Diabetic Retinopathy. In this condition, the capillaries of retina shut down. This causes new blood vessel to grow in retina [1].

In order to study eye diseases like Diabetic Retinopathy ophthalmologists uses different retinal images, such retinal images are usually called as fundus images. One of the fundus image labeled, with various feature components of Diabetic Retinopathy is shown in Fig. 1. Microaneurysms are small saccular pouches caused by local distension of capillary walls and seen as small red dots. This may also lead to big blood clots called hemorrhage. Hard Exudates are yellow lipid deposits which appear as bright yellow lesions. The bright circular region from where the blood vessels emanate is called as optic disk. The fovea defines the center of the retina, and is the region of highest visual acuity [2]. For early detection of DR, detection of Hard Exudates plays an important role.



Fig. 1. Illustration of various features of a typical Retinopathy image

Over the past few years many techniques for detection of Diabetic Retinopathy are given in literature. V. Vijaya Kumari et al [1] uses mathematical morphology for extracting Exudates, optic disk and blood vessels. Here, Morphological opening and closing operations of different sizes are used to detect Hard Exudates, but one of the main drawbacks of morphological operation is the size of structuring element suitable for one image is not be suitable for another image. Saiprasad Ravishankar et al [2] also uses mathematical morphology to detect hard Exudates but it uses a linear classifier for classifying the patches based on their edge strength. Hussain F. Jaafar et al [3] introduced a pure splitting technique for detection of Exudates in retinal images. This technique uses an adaptive thresholding based on a novel algorithm for pure splitting of the image. S. Kavitha et al [4] uses mathematical morphology and pure splitting technique to detect faint Exudates. Sopharak et al [5] used a Naive Bayes classifier to detect Exudates. The main drawback of Naive Bayes classifier is it misses the faint Exudates. R. Vijayamadheswaran et al [6] proposed detection of Exudates using a combination of Contextual Clustering and Radial basis function. In this technique all the fundus images are transformed to a standard template image condition. Detection of Exudates is accurate when the fundus images are captured with good quality. Garcia et al [7] detects Hard Exudates using neural network (NN) approach. Here Three NN classifiers were investigated which includes multilayer perceptron (MLP), radial basis function (RBF) and support vector machine (SVM). This paper presents two techniques for detection of hard Exudates. The first technique discusses a modified 
approach based on mathematical morphology. In the second technique a hybrid approach for detection of Hard Exudates is proposed. Here, mathematical morphology is combined with clustering approach to improve the accuracy of Exudates detection.

In this paper, a publicly available DIARETDB1 database is used to verify the result[8]. This Database is publicly available and it has the ground truth collected from several experts. The database consists of 89 color fundus images out of which 84 contain at least mild non-proliferative signs of the Diabetic Retinopathy and 5 are considered normal that do not contain any signs of the Diabetic Retinopathy according to all experts participated in the evaluation. The images were taken in the Kuopio University Hospital. It also has a strict evaluation protocol. The protocol is demonstrated with a baseline method included in the available tool kit. This study provides the means for the reliable evaluation of automatic methods for detecting Diabetic Retinopathy. In addition to this, the fundus images related to the Diabetic Retinopathy are evaluated by using sensitivity and specificity. Sensitivity is the percentage of abnormal fundus images classified as abnormal, and specificity is the percentage of normal funds classified as normal by the screening. The higher the sensitivity and specificity values, the better the diagnosis [8].

This paper is composed of five sections. Section II discusses Modified Morphology based Exudates detection. Section III presents the proposed method for Exudates detection. In section IV, Experimental Results are presented while Conclusion is included in Section V.

\section{Modified MATHEMATICAL MoRPHOlOGY BASED HARD EXUDATES DETECTION}

Mathematical Morphology is a theory and technique for analysis and processing of geometrical structures (such as size, shape, and convexity), based on set theory, lattice theory, topology, and random functions [9]. The technique used here is a modification of morphology based technique given by Vijaya Kumari et al [1]. For detection of Hard Exudates three steps applied are extraction of the green channel, morphological processing, optic disk detection and removal.

\section{A. Extraction of Green Channel}

Here, Input image is initially resized to a standard size of $768 \times 576$ pixels. Resized image is RGB image. So, from resized image we extract only green channel image. We select the green channel because fundus images are almost always saturated in the red channel and have very low contrast in the blue channel [2].

\section{B. Morphological processing}

Exudates are the primary sign of Diabetic Retinopathy. Initially dilation is performed on the green channel extracted image across two disc shape structuring elements. Dilation is an operation that grows or thickens object in a binary image [1]. It is defined in terms of set operation as given by (1).

$$
\mathrm{A} \oplus \mathrm{B}=\mathrm{A}_{1}(\mathrm{X}, \mathrm{Y})=\max _{\mathrm{i}, \mathrm{j} \varepsilon \mathrm{B}}(\mathrm{A}(\mathrm{X}-\mathrm{i}, \mathrm{Y}-\mathrm{j})+\mathrm{B}(\mathrm{i}, \mathrm{j}))
$$

Here, $\mathrm{A}$ is an input image, $\mathrm{B}$ is the structuring element used for dilation.

Once the Image is dilated across two structuring elements both the results are subtracted to get the boundary of Hard Exudates. In order to get exact boundaries the image is thresholded using Otsu's thresholding technique. Then Morphological filling is done to get the closed regions. Once closed regions are obtained, morphological opening is performed to remove small bright details. Thus, the regions which are possible candidates for Hard Exudates are obtained in this step.

\section{Optic Disk Detection and removal}

In the morphological processing step the regions corresponding to possible Hard Exudates are obtained. But it was observed, that normally Hard Exudates and Optic disk are of the same brightness, color and contrast [1]. Hence, optic disk is sometimes misclassified as Hard Exudates. Therefore, Optic disk must be eliminated from the possible candidates. Here, Circular Hough Transform is applied to detect the optic disk. Once detected, it is eliminated and the Hard Exudate regions are obtained.

\section{PROPOSED ALGORITHM}

The proposed Algorithm consists of three main stages, Preprocessing, Clustering and Post processing.

\section{A. Preprocessing}

The main purpose of this stage is to remove unwanted details from an image and make the image suitable for applying next step. Here, Input image is initially resized to fixed size of $256 \times 256$. Green channel is extracted from resized image and then morphological dilation is applied by using disk shape structuring element.

\section{B. Clustering}

Once the image is dilated, clustering algorithm is applied on image to get Hard Exudates. Here two different types of clustering algorithms are implemented.

\section{1) Linde - Buzo - Gray Algorithm}

The LBG Algorithm is an iterative clustering technique. For the purpose of explaining this algorithm, two dimensional vector space as shown in Fig. 2 is considered. In this figure each point represents two consecutive pixels. In this algorithm centroid is computed as the first code vector $\mathrm{C} 1$ for the training set. In Fig. 2 two vectors v1 \& v2 are generated by adding constant error to the code vector. Euclidean distances of all the training vectors are computed with vectors v1 \& v2 and two clusters are formed based on nearest of v1 or v2. The procedure is repeated for these two clusters to generate four new clusters. This procedure is repeated for every new cluster until the required size of the codebook is reached [10]. 


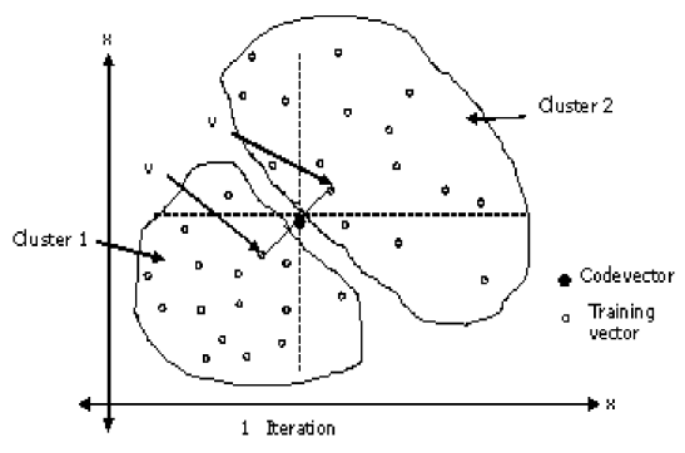

Fig. 2. LBG for two dimensional case

Modified LBG Algorithm derived from [10] is explained as follows

1. Start.

2. Read the input image A.

3. Resize image A to a fixed size of $256 \times 256$.

4. Divide the image into two $2 \times 2$ non overlapping blocks.

5. Represent each block in the form of training vector space. Each block is converted to the training vector $\mathrm{X}_{\mathrm{i}}=\left(\mathrm{x}_{\mathrm{i} 1}, \mathrm{x}_{\mathrm{i} 2}, \mathrm{x}_{\mathrm{i}}\right)$.

6. Consider entire training vector space as one cluster.

7. Find the centroid of the cluster. i.e. code vector of codebook.

8. Initialize error vector (E) which will be of the same size as codevector generated in step 7.

9. Add and subtract constant vector i.e. error vector from codevector generated in step 7 and get two new vectors as follows:

$\mathrm{C} 1=\mathrm{C}+\mathrm{E}$

$\mathrm{C} 2=\mathrm{C}-\mathrm{E}$

10. Find Euclidean distance by considering each training vector in training vector space with respect to $\mathrm{C} 1$ and $\mathrm{C} 2$ as follows:

$$
\mathrm{d}\left(\mathrm{X}_{\mathrm{i}}, \mathrm{C}_{\mathrm{j}}\right)=\sum_{\mathrm{p}=1}^{\mathrm{k}}\left(\mathrm{x}_{\mathrm{ip}}-\mathrm{c}_{\mathrm{jp}}\right)^{2}
$$

where,

$\mathrm{X}_{\mathrm{i}}$ is the training vector,

$\mathrm{C}_{\mathrm{j}}$ is the codevector of the codebook

11. If training vector is closed to $\mathrm{C} 1$ put it in cluster 1 else in cluster 2 and form clusters.

12. Repeat step 8 to 11 until codebook of the desired size is obtained.

13. Stop.

2) k-means Algorithm

$k$-means is an unsupervised clustering algorithm which is used to classify input image to $k$ clusters based on the nearest mean.
The modified algorithm for $k$-means derived from [11] is explained as follows

1. Start.

2. Read the input image A.

3. Resize image A to a fixed size of $256 \times 256$.

4. Divide the image into two $2 \times 2$ non overlapping blocks.

5. Represent each block in the form of a training vector space $X$. Each block is converted to the training vector $\mathrm{X}_{\mathrm{i}}=\left(\mathrm{x}_{\mathrm{i} 1}, \mathrm{x}_{\mathrm{i} 2}, \mathrm{x}_{\mathrm{ik}}\right)$.

6. Select $k$ random vectors from the training set and call it as initial codebook $\mathrm{C}$.

7. Select training vector $X_{i}$ from training vector space.

8. Calculate squared Euclidean distance of $X_{i}$ with the codebook $\mathrm{C}$ as follows:

$\mathrm{d}\left(\mathrm{X}_{\mathrm{i}}, \mathrm{C}_{\mathrm{j}}\right)=\sum_{\mathrm{p}=1}^{\mathrm{k}}\left(\mathrm{x}_{\mathrm{ip}}-\mathrm{c}_{\mathrm{jp}}\right)^{2}$

where,

$\mathrm{X}_{\mathrm{i}}$ is the training vector,

$\mathrm{C}_{\mathrm{j}}$ is the codevector of the codebook

9. Calculate value of minimum Euclidian distance as follows:

$$
\mathrm{d}\left(\mathrm{X}_{\mathrm{i}}, \mathrm{C}_{\min }\right)=\min _{\mathrm{i} \leq j \leq \mathrm{N}}\left\{\mathrm{d}\left(\mathrm{X}_{\mathrm{i}}, \mathrm{C}_{\mathrm{j}}\right)\right\}
$$

10. Insert $X_{\mathrm{i}}$ vector to cluster corresponding to $C_{\min }$.

11. Repeat steps 7 to 10 for all training vectors in training vector space.

12. Compute the centroid for each cluster by taking column wise average of each codevector in the codebook.

13. Generate the codebook by inserting the centroids obtained in step 12 and give it as input to the next iteration.

14. Repeat steps 7 to 13 until codebook obtained in successive two iterations are same.

15. Stop.

\section{Post processing}

Once clustering Algorithm is applied to Dilated Image, Hard Exudates Regions are obtained in any of the one cluster from $k$ clusters. It has been observed that normally Hard Exudates and Optic disk are of same brightness, color and contrast.

So, sometimes optic disk is misclassified and must be eliminated from the Hard Exudates result obtained after applying a clustering algorithm to get accurate Hard Exudates. Here, Circular Hough Transform is applied to the result obtained by applying a clustering algorithm for detecting optic disk. Once the optic disk is detected it is eliminated and the Hard Exudates Result is obtained. 


\section{RESUlT AND DisCUSSION}

For Evaluation of proposed technique we have used 89 Images provided in DIARETDB1 Database. This database is selected because this database is publicly available and it has the ground truth collected from several experts. This ground truth acts as a good mean for verifying the results of the proposed algorithm with the findings of ophthalmologists provided in DIARETDB1 database. All 89 Images were tested on Intel Core i5-2410M, 3GB RAM using Matlab R2011b. Image results were obtained in approximately 7 seconds. To evaluate performance of our proposed Algorithm values of the three parameters are calculated which includes Sensitivity, Specificity and Accuracy [12]. These parameters are calculated by using (2), (3) and (4) .

$$
\begin{aligned}
& \text { Sensitivity }=\frac{T P}{T P+F N} \\
& \text { Specificity }=\frac{T N}{T N+F P} \\
& \text { Accuracy }=\frac{(T P+T N)}{(T P+F P+T N+F N)}
\end{aligned}
$$

where, TP (true positive) is number of pixels classified as Exudates by both the ophthalmologist and the algorithm, FP (false positive) is number of non-exudates pixels which are wrongly detected as Exudates pixels by the algorithm, TN (True negative) is number of no Exudates pixels which are identified as non-Exudates pixels by both the ophthalmologist and the algorithm, FN (false negative) is number of Exudates pixels that are not detected by the algorithm but are considered as Exudates by ophthalmologist.

The result is optimal for highest sensitivity, sensitivity and accuracy's value. Initially Hard Exudates detection was done using modified morphological approach. The result of Exudates detection using mathematical morphology is as shown in Fig. 3. After implementing morphology based detection technique it is observed that out of three evaluation parameters the value of specificity and accuracy is less in this case. Hence same database images were tested using LBG Algorithm. The result of Exudates detection using LBG is as shown in Fig. 4. In this case it is observed that the value of specificity was increased as compared to morphology based approach but the value of sensitivity was largely reduced. So, $k$-means algorithm was implemented. Result of $k$-means is as shown in Fig. 5. Here, the result obtained is good as compare to morphology based detection and LBG.

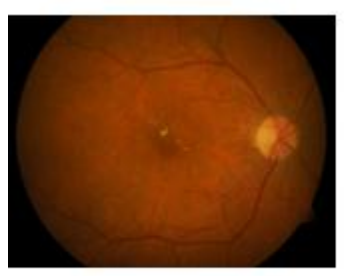

(a)

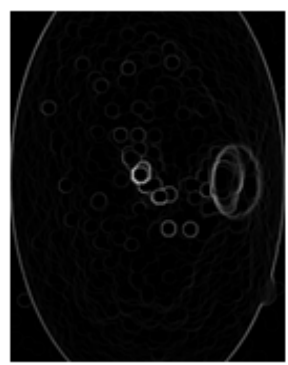

(e)

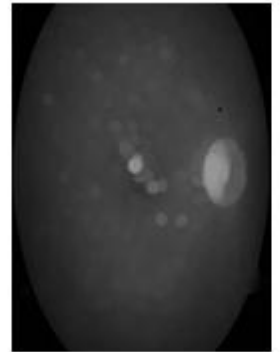

(b)

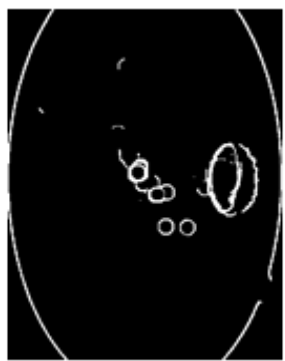

(f)

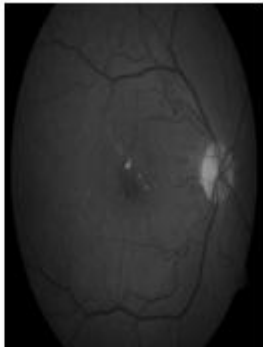

(c)

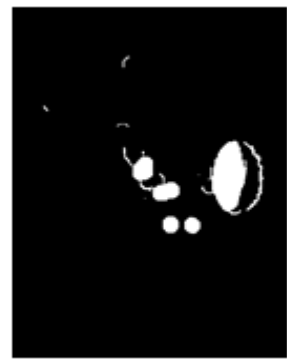

(g)

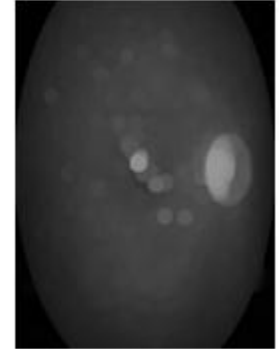

(d)



(h)

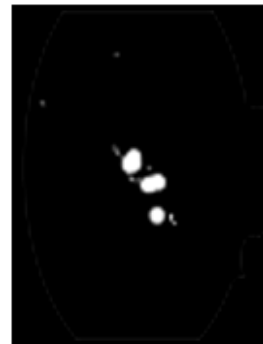

(i)

Fig. 3. Hard Exudates using Morphology (a) Original Image (b) Green Channel Extracted Result (c) Dilated Image1 (d) Dilated Image2 (e) Subtracted Image (f) Thresholded Result (g) Filled Image (h) Result after Open (i) Final Hard Exudates 
Summary of results obtained after applying all the three algorithms is as shown in Table I. From Table I it is clear that $k$-means gives better result as compared to other two techniques.

One of the main parameters in clustering algorithm is choosing number of clusters which gives best result. Hence, here DIARETDB1 database is tested by considering the number of clusters as 4, 6 and 8 in $k$-means algorithm as shown in Fig. 6.
It is observed that $k$-means with 8 clusters gives optimal results. Same Database is also tested by LBG algorithm with the number of clusters as 4 and 8 as shown in Fig. 7. Here, it is observed that for LBG best results are obtained when the number of clusters is chosen as 8 . For both $k$-means and LBG clustering when the number of clusters increased more than 8 the Exudates were split into different clusters. Hence, the results are not accurate for more than 8 clusters.

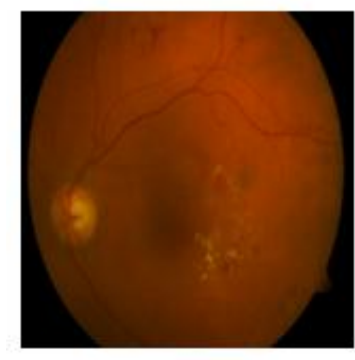

(a)

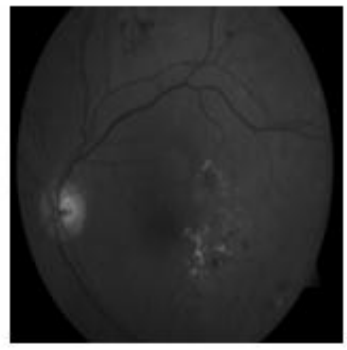

(b)

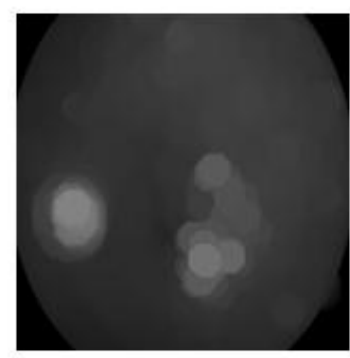

(c)

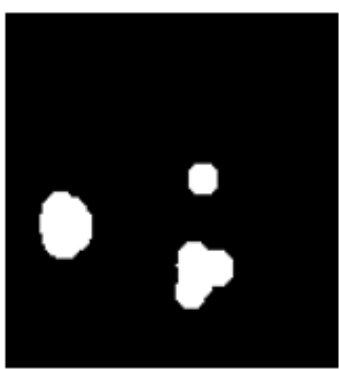

(d)

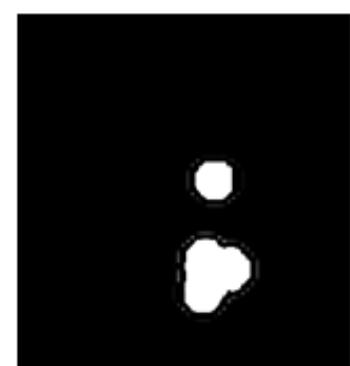

(e)

Fig. 4. Hard Exudates using LBG (a) Original Image (b) Green Channel Extracted Result (c) Dilated Image (d) LBG Result (e) Hard Exudates

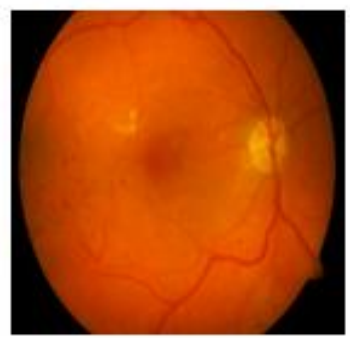

(a)

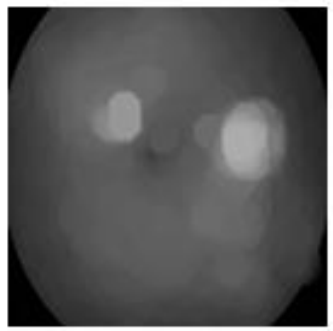

(b)

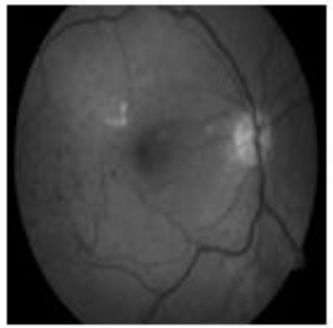

(c)

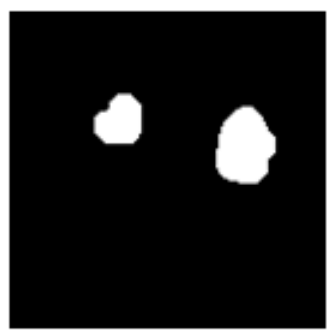

(d)

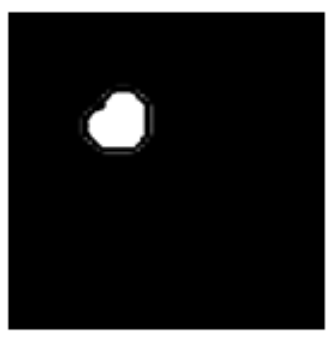

(e)

Fig. 5. Hard Exudates using $k$-means (a) Original Image (b) Green Channel Extracted Result (c) Dilated Image (d) $k$-means Result (e) Hard Exudates 
TABLE I. COMPARISION OF HARD EXUDATES RESULT

\begin{tabular}{|c|c|c|c|}
\hline \multirow{2}{*}{ Algorithm } & \multicolumn{3}{|c|}{ Evaluation Parameters } \\
\cline { 2 - 4 } & Sensitivity & Specificity & Accuracy \\
\hline $\begin{array}{c}\text { Morphology } \\
\text { Based } \\
\text { Approach }\end{array}$ & $91 \%$ & $39 \%$ & $67 \%$ \\
\hline LBG & $80 \%$ & $57 \%$ & $68 \%$ \\
\hline$k$-means & $77 \%$ & $76 \%$ & $76 \%$ \\
\hline
\end{tabular}

\begin{tabular}{|c|c|c|c|}
\hline \multicolumn{4}{|c|}{ Optimal value of number of clusters } \\
\hline \multicolumn{4}{|l|}{$=90$} \\
\hline \multirow{2}{*}{ 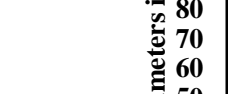 } & \multicolumn{3}{|c|}{$\mathrm{r}$} \\
\hline & \multicolumn{3}{|c|}{ 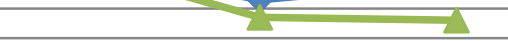 } \\
\hline \multirow{3}{*}{ 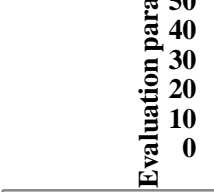 } & & & \\
\hline & & & \\
\hline & 8 cluster & 6 cluster & 4 cluster \\
\hline$\approx$ Acuuracy & 76 & 63 & 69 \\
\hline- Sensitivity & 77 & 69 & 81 \\
\hline- Specificity & 76 & 57 & 56 \\
\hline
\end{tabular}

Fig. 6. Comparison Results for optimal value of number of clusters $k$ in $k$-means



Fig. 7. Comparison Results for optimal value of number of clusters $k$ in LBG

\section{CONCLUSION}

The main idea of detection of Diabetic Retinopathy is to detect Hard Exudates. In this paper, a fast method for detection of Hard Exudates is proposed. This method consists of three stages in the preprocessing stage image is brought to the form where clustering technique yields accurate results Second stage is clustering. Here, $\mathrm{LBG}$ and $k$-means clustering techniques are applied to detect Hard Exudates and third stage is Post processing where Optic Disk is removed from clustering results to get accurate Hard Exudates. The final results obtained from these techniques are compared with the results obtained from Morphology based detection and the ground truth provided in DIARETDB1 database. The performance of Algorithm is evaluated by calculating values of sensitivity, specificity and accuracy. Our result suggests that clustering using $k$-means produce best result as compared to other two techniques.

\section{REFERENCES}

[1] Vijaya Kumari, N. Suriyanarayanan, C.Thanka Saranya , "A Feature Extraction for Early Detection of Diabetic", International Conference on Recent Trends in Information Telecommunication and Computing, pp. $359-361,2010$.

[2] Saiprasad Ravishankar, Arpit Jain, Anurag Mittal, "Automatic Feature Extraction for Detection of Diabetic Retinopathy in Fundus Images", International Conference on Computer Vision and Pattern Recognition, pp. 210-217, 2009.

[3] Hussain F. Jaafar, Asoke K. Nandi and Waleed Al-Nuaimy, "Detection of Exudates in Retinal Images Using a Pure Splitting Technique", 32nd Annual International Conference of the IEEE EMBS Buenos Aires, Argentina, pp. 6745 - 6748, 2010.

[4] S. Kavitha, K. Duraiswamy, A. R. S. Sri Supreetha, "Detection of Exudates and macula in fundus images to estimate severity of Diabetic Retinopathy", International Journal of Communications and Engineering Vol.7, Issue: 01, pp. 24-29, March 2012.

[5] Sopharak, Akara, Dailey, Matthew N., Uyyanonvara, Bunyarit, Barman, Sarah, Williamson, Tom, Nwe, Khine Thet and Moe, Yin Aye, "Machine learning approach to automatic exudate detection in retinal images from Diabetic Patients", Journal of Modern Optics, Vol. 5, Issue 2, pp. 124-135, 2010.

[6] R. Vijayamadheswaran, Dr. M. Arthanari, Mr. M. Sivakumar, "Detection of Diabetic Retinopathy using Radial Basis Function", International journal of Innovative technology \& creative Engineering, Vol.1, pp. 41-47, January 2011.

[7] María García, Clara I. Sánchez, María I. López, Daniel Abásolo, and Roberto Hornero, "Neural Network based detection of Hard Exudates in Retinal Images", Computer Methods and Programs in Biomedicine, Vol.93, Issue 1, pp. 9-19, January 2009.

[8] Tomi Kauppi, Valentina Kalesnykiene, Joni-Kristian Kamarainen, Lasse Lensu, liris Sorri, Asta Raninen, Raija Voutilainen, Hannu Uusitalo, Heikki K"alvi"ainen and Juhani Pietila, "DIARETDB1 Diabetic Retinopathy database and evaluation protocol", Technical report, Faculty of medicine, University of Kuopio, Finland, 2007.

[9] Ardimas Andi Purwita, Kresno Adityowibowo, Ashlih Dameitry, Made Widhi Surya Atman, "Automated Microaneurysm Detection Using Mathematical Morphology", International Conference on Instrumentation, Communication, Information Technology and Biomedical Engineering , pp. 117 - 120, November 2011.

[10] Dr. H.B. Kekre, Tanuja K. Sarode, Suchitra M. Patil, "2D Image Morphing With Wrapping Using Vector Quantization Based Colour Transition", International Journal of Computer Science and Information Security, Vol. 9, No. 7, pp. 75-82, 2011.

[11] Dr. H.B. Kekre, Tanuja K. Sarode, "Vector Quantized Codebook Optimization using K-Means", International Journal on Computer Science and Engineering, Vol.1, No. 3, pp. 283-290, 2009.

[12] John Attia, "Moving Beyond Sensitivity And Specificity: Using Likelihood Ratios To Help Interpret Diagnostic Tests". Austral. Prescriber, Vol.26, No.5, pp. 111-113, 2003. 